\title{
A three-microRNA signature for lung squamous cell carcinoma diagnosis in Chinese male patients
}

\author{
Lan Zhang ${ }^{1, *}$, Xia Shan ${ }^{2, *}$, Jun Wang ${ }^{3, *}$, Jun Zhu ${ }^{4}$, Zebo Huang ${ }^{1}$, Huo Zhang ${ }^{1}$, Xin \\ Zhou $^{1}$, Wenfang Cheng ${ }^{5}$, Yongqian Shu ${ }^{1}$, Wei Zhu ${ }^{1}$ and Ping Liu ${ }^{1}$ \\ ${ }^{1}$ Department of Oncology, First Affiliated Hospital of Nanjing Medical University, Nanjing 210029, PR China \\ ${ }^{2}$ Department of Respiration, The Affiliated Jiangning Hospital of Nanjing Medical University, Nanjing 210000, PR China \\ ${ }^{3}$ Department of Thoracic Surgery, First Affiliated Hospital of Nanjing Medical University, Nanjing 210029, PR China \\ ${ }^{4}$ Department of Radiation Oncology, Jiangsu Cancer Hospital, Nanjing 210009, PR China \\ ${ }^{5}$ Department of Gastroenterology, First Affiliated Hospital of Nanjing Medical University, Nanjing 210029, PR China \\ *These authors have contributed equally to this work \\ Correspondence to: Wei Zhu, email: zhuwei@njmu.edu.cn \\ Ping Liu, email: liupinga28@163.com \\ Keywords: serum microRNA, lung SCC, diagnostic biomarker, GRT-PCR \\ Received: December 21, $2016 \quad$ Accepted:May 16, $2017 \quad$ Published: July 28, 2017 \\ Copyright: Zhang et al. This is an open-access article distributed under the terms of the Creative Commons Attribution License 3.0 \\ (CC BY 3.0), which permits unrestricted use, distribution, and reproduction in any medium, provided the original author and source \\ are credited.
}

\section{ABSTRACT}

Various studies have demonstrated the diagnostic value of microRNA (miRNA) for lung cancer, but miRNA signatures varied between different subtypes. Whether serum miRNAs could be used as biomarkers in lung squamous cell carcinoma (SCC) remains unknown. Using quantitative real-time polymerase chain reaction (qRT-PCR) based Exiqon panel, 38 differentially expressed miRNAs were identified from 3 male lung SCC pool samples and 1 normal control (NC) pool in the initial screening phase. After the training (24 SCC VS. 15 NCs), testing (44 SCC VS. 57 NCs) and external validation ( 34 SCC VS. 36 NCs VS. 10 pulmonary hamartoma) processes via qRTPCR, we identified a three-miRNA panel ( (miR-106a-5p, miR-20a-5p and miR-93$5 p)$ to be a potential diagnostic marker for male lung SCC patients. The areas under the receiver operating characteristic (ROC) curve of the three-miRNA panel for the training, testing and validation phases were $0.969,0.881$ and 0.954 respectively. In addition, this signature could also differentiate lung SCC from pulmonary hamartoma (AUC=0.900). The 3 miRNAs were consistently up-regulated in lung SCC tissues (23 SCC VS. 23 NCs) and serum exosomes (17 SCC VS. 24 NCs). Moreover, expression of the 3 miRNAs was decreased in arterial serum $(n=3)$. In conclusion, we established a three-miRNA signature in the peripheral serum with considerable clinical value in the diagnosis of male lung SCC patients.

\section{INTRODUCTION}

Lung cancer is the leading cause of cancer mortality in both men and women worldwide [1]. Non-small cell lung cancer (NSCLC) accounts for approximately 85\% of all lung cancers. Among NSCLCs, squamous cell carcinoma (SCC) is one of the major histological subtypes [2]. The majority of lung SCC patients is male and had a smoking history [3, 4]. Although surgical resection shows promise in treating lung SCC, a lack of effective tools for early diagnose leads to low 5-year survival rates [2]. Low-dose computed tomography (LDCT) has been explored as a method for the diagnosis of early lung SCC, however, it still has several limitations [5]. None-invasive markers such as squamous cell carcinoma antigen (SCC $\mathrm{Ag}$ ), neuron-specific enolase (NSE) and Cyfra 21-1 are not sensitive and specific enough for the routine use in the diagnose of lung SCC [6]. Thus, new and non-invasive biomarkers with high diagnostic power for lung SCC patients are urgently needed. 
Circulating miRNAs have emerged as reliable noninvasive biomarkers for the early diagnosis of cancer [7, 8]. Specifically, the expression patterns of human serum miRNAs have been reported to have the potential to identify various types of cancers [9-12]. These findings suggest that there might also be a unique serum miRNA expression signature that could distinguish lung SCC patients with normal individuals. Male accounts for more than $90 \%$ of lung SCC patients and have different biological behavior and treatment response compared with female patients [2]. So it is of great significance to discover a unique miRNA signature for the diagnosis of male lung SCC patients. However, unlike the assessment of cellular miRNA levels for which there are accepted housekeeping genes, it still remains unclear whether any circulating miRNAs have prototypical housekeeping functions or could be present at sufficiently stable levels to serve as effective reference controls.

In the present study, using both miRCURY platform and quantitative real-time polymerase chain reaction (qRTPCR), we sought to identify a panel of serum miRNAs that could serve as a novel biomarker for the diagnosis of male lung SCC patients. The expression profile of selected miRNAs was then assessed in tissue and serum exosomes to explore the origin and potential form in circulation. Finally, we compared miRNA expression in peripheral venous and arterial serum.

\section{RESULTS}

\section{Identification of stable reference miRNAs in human serum samples}

The potential reference miRNAs (miR-16-5p [13, 14], miR-103a-3p [15, 16], U6 [11, 16] and miR-191$5 p[11,17])$ examined in present study were selected primarily because they had relatively stable expression level according to previous studies. In geNorm, high variation in expression elevates $M$ values and indicates low stability, whereas low $\mathrm{M}$ values indicate high expression stability [16, 18]. In human serum samples, miR-16$5 p$ and miR-103a-3p both showed the lowest $M$ value (Supplementary Figure 1). In addition, miR-16-5p might be more stable than miR-103a-3p because of the lower $\mathrm{Ct}$ value compared with miR-103a-3p (Supplementary Table 2). Therefore, miR-16-5p was selected as the internal reference control in the present study.

\section{Patient description}

Serum samples from male lung SCC patients and NCs were randomly assigned to 3 independent sets: training stage, testing stage and external validation stage (the flow chart was shown in Figure 1). The clinical and demographics features of the lung SCC patients and NCs are listed in Table 1. There were no significant differences in the distribution of age, smoking and sex between the cancer patients and NCs.

\section{Discovery of candidate miRNAs from pooled serum samples}

A total of 168 miRNAs were sequenced by the Exiqon miRCURY-Ready-to-Use PCR-Human-panelI+II-V1.M based on the qRT-PCR platform between in 3 peripheral serum pools from 30 male lung SCC patients and 1 pooled sample from $10 \mathrm{NCs}$. Candidate miRNAs were selected based on the following criteria: (a) with a $\mathrm{Ct}$ value $<37$; (b) exhibiting $5 \mathrm{Ct}$ lower than negative control (No Template Control, NTC); (c) having at least a 1.5-fold altered expression. Levels of 26 miRNAs were significantly higher in male lung SCC patients than controls. In contrast, levels of 12 miRNAs were significantly lower (Supplementary Table 1). Taken together, 38 differentially expressed miRNAs were selected as candidate miRNAs and were chosen to be further assessed via qRT-PCR.

\section{Confirmation of candidate miRNAs by qRT-PCR analysis}

Our study first tested the 38 candidate miRNAs in the training stage including 24 male lung SCC patients and 15 NCs. A total of 14 miRNAs demonstrated differential expression and were validate in the testing stage (Supplementary Table 1). In the larger cohort, 3 of the 14 miRNAs (miR-106a-5p, miR-20a-5p and miR-93$5 \mathrm{p})$ showed consistent up-regulation. To verify the three miRNAs as a signature for male lung SCC patients, the expression of the three miRNAs was further assessed using an additional independent cohort of 34 lung SCC patients and $36 \mathrm{NCs}$. The expression trend of the three miRNAs was consistent with the training set and the testing stages, with all the miRNAs were upregulated $>$ 2-fold (Supplementary Table 2). As a result of our multiphase analysis, we identified a three-miRNA panel to be the potential signature for the detection of male lung SCC patients (Figure 2).

\section{Diagnostic value of the three-miRNA signature in serum}

To evaluate the diagnostic value of this three-miRNA signature in discriminating male lung SCC patients from NCs, ROC curve analysis was performed. The AUCs were 0.834 (95\% confidence interval (CI):0.781-0.887), 0.804 (95\% CI:0.746-0.863), 0.823 (95\% CI:0.767-0.879) respectively (Supplementary Figure 2). Moreover, when the three miRNAs were combined together as a panel, the AUCs were 0.832 (95\% CI: 0.780-0.885; Figure 3a). Meanwhile, the diagnostic performance of the threemiRNA panel was also assessed in the training, testing and 


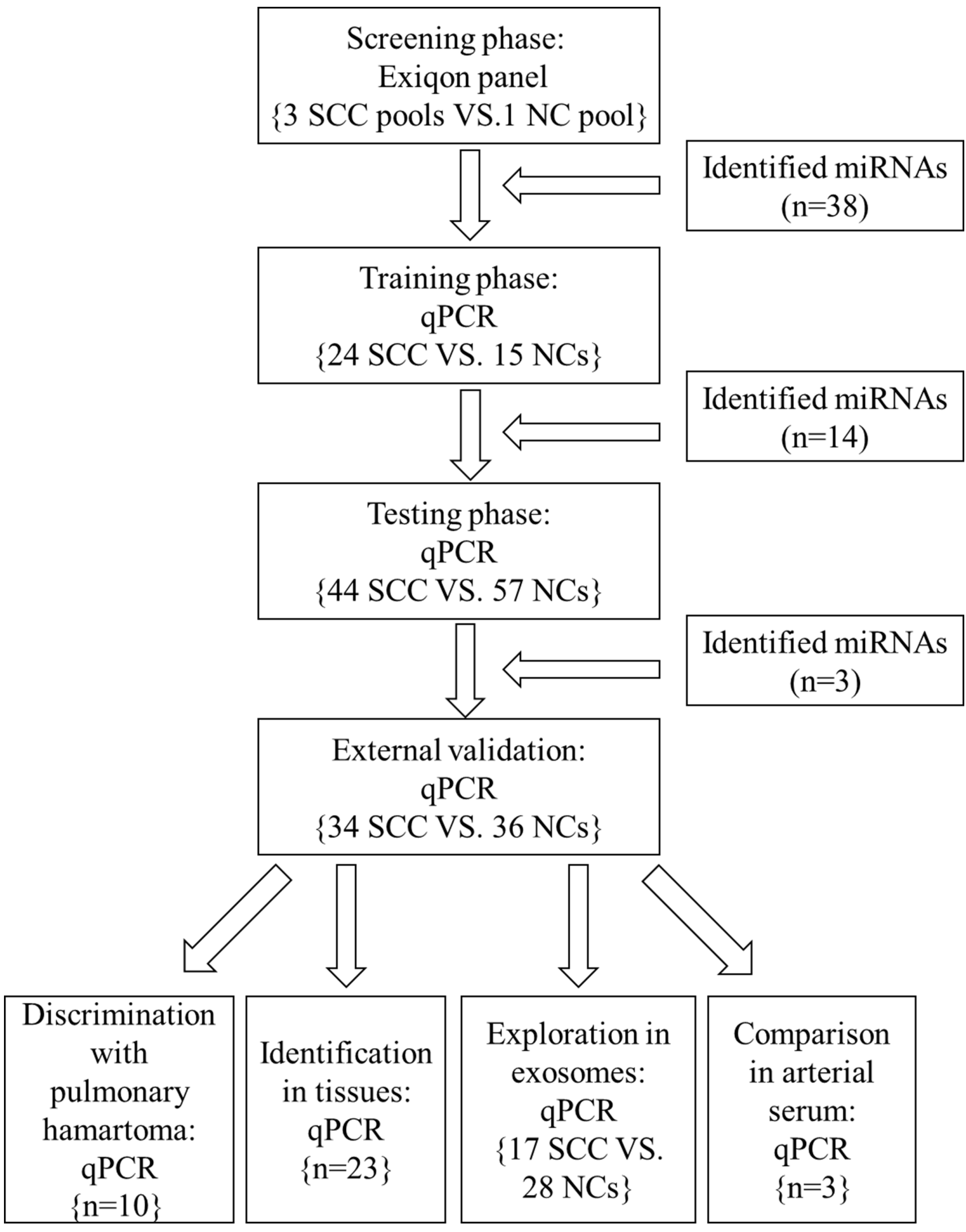

Figure 1: The flow chart of the experiment design. SCC: squamous cell carcinoma; NC: normal control. 
Table 1: Characteristics of 102 SCC patients and 108 normal controls enrolled in the study

\begin{tabular}{|c|c|c|c|c|c|c|c|c|}
\hline \multirow[t]{2}{*}{ Variables } & \multicolumn{2}{|c|}{$\begin{array}{l}\text { Screening phase } \\
\qquad(n=40)\end{array}$} & \multicolumn{2}{|c|}{$\begin{array}{l}\text { Training stage } \\
\qquad(n=39)\end{array}$} & \multicolumn{2}{|c|}{$\begin{array}{l}\text { Testing stage } \\
\quad(n=101)\end{array}$} & \multicolumn{2}{|c|}{$\begin{array}{l}\text { External validation stage } \\
\qquad(n=70)\end{array}$} \\
\hline & Case $(\%)$ & Controls (\%) & Case (\%) & Controls (\%) & Case (\%) & Controls (\%) & Case (\%) & Controls (\%) \\
\hline Number & 30 & 10 & 24 & 15 & 44 & 57 & 34 & 36 \\
\hline \multicolumn{9}{|l|}{ Age } \\
\hline$<65$ & $19(63.3)$ & $6(60.0)$ & $15(62.5)$ & $9(60.0)$ & $25(56.8)$ & $32(56.1)$ & $20(58.8)$ & $19(52.8)$ \\
\hline$\geq 65$ & $11(36.7)$ & $4(40.0)$ & $9(37.5)$ & $6(40.0)$ & $19(43.2)$ & $25(43.9)$ & $14(41.2)$ & $17(47.2)$ \\
\hline \multicolumn{9}{|c|}{ Smoking history } \\
\hline Former & $6(20.0)$ & $3(30.0)$ & $3(12.5)$ & $2(13.3)$ & $5(11.3)$ & $8(14.0)$ & $3(8.80)$ & $3(8.80)$ \\
\hline Current & $24(80.0)$ & $7(70.0)$ & $21(87.5)$ & $13(86.7)$ & $39(88.7)$ & $49(86.0)$ & $31(91.2)$ & $33(91.2)$ \\
\hline \multicolumn{9}{|l|}{ TNM stage } \\
\hline I & $6(20.0)$ & & $8(33.3)$ & & $18(40.9)$ & & $14(41.2)$ & \\
\hline II & $17(56.7)$ & & $13(54.2)$ & & $21(47.7)$ & & $12(35.3)$ & \\
\hline III & $7(23.3)$ & & $3(12.5)$ & & $5(11.4)$ & & $8(23.5)$ & \\
\hline \multicolumn{9}{|l|}{ Differentiation } \\
\hline Well & $3(10.0)$ & & $3(12.5)$ & & $5(11.4)$ & & $3(8.80)$ & \\
\hline Moderately & $18(60.0)$ & & $12(50.0)$ & & $21(47.7)$ & & $18(52.9)$ & \\
\hline Poorly & $9(30.0)$ & & $9(37.5)$ & & $18(40.9)$ & & $13(38.3)$ & \\
\hline
\end{tabular}

external validation stages separately and the AUCs were 0.969 (95\% CI: 0.924-1.000; Figure 3b), 0.881 (95\% CI: $0.810-0.953$; Figure $3 \mathrm{c}$ ) and 0.954 (95\% CI: 0.910-0.998; Figure $3 \mathrm{~d}$ ) respectively.

In addition, the diagnostic performance of the established miRNA panel was also analyzed at different TNM stage. The corresponding AUCs for lung SCC patients with TNM stage I, II, III were 0.904, 0.862 and 0.882 respectively (Supplementary Figure 3a-3c).

Our study also evaluated the diagnostic value of the three-miRNA panel in discriminating lung SCC from pulmonary hamartoma in the external validation stage. The result showed that the miRNA panel possessed high accuracy in discriminating lung SCC from pulmonary hamartoma $\quad(\mathrm{AUC}=0.900 ; \quad 95 \% \quad \mathrm{CI}: \quad 0.804-0.996$; Supplementary Figure 3d).

Finally, the association of the three serum miRNAs with clinical stage was also evaluated. However, none of the three miRNAs demonstrated significant difference in patients with stage III compared to those with stage I+II (data not shown).

\section{Identification of miRNA expression in tissue samples}

The expression levels of the three miRNAs were examined in 23 pairs of tissue samples of lung SCC patients. As it is demonstrated in Figure 4, the expression of miR-106a-5p, miR-20a-5p and miR-93-5p was significantly higher in tumor tissue samples than the NCs, which was consistent with the result obtained from serum samples.

\section{Exploration of miRNA expression in serum exosomes}

To further explore the potential form of the three identified miRNAs in serum, esosomal miRNA expression extracted from 17 lung SCC and 28 NC serum samples were examined. As it is shown in Figure 5, the expression of the three miRNAs was all up regulated with statistical significance.

\section{Comparison of miRNA expression in venous with arterial serum}

To discover the difference of miRNA expression between venous and arterial serum, another three pairs of samples were examined. In arterial serum, the expression levels of miR-106a-5p, miR-20a-5p and miR-93-5p were all decreased and yielded borderline statistical significance $(P=0.083,0.066$ and 0.071 respectively) (Supplementary Figure 4). 


\section{Bioinformatics analysis of miR-106a-5p, miR- 20a-5p and miR-93-5p}

The putative target genes of miR-106a-5p, miR20a-5p and miR-93-5p were identified by DIANATarBase v7.0. Then DIANA-miRPath v3.0 was utilized for KEGG pathway analysis (Supplementary Table 4) and GO category analysis (Supplementary Table 5) to investigate the pathways significantly associated with the three miRNAs. All of the three miRNAs are related to pathological mechanisms of cancer such as by interacting with MAPK and mTOR signaling.

\section{DISCUSSION}

In present study, we established a carefully designed procedure to identify a serum miRNA profile of lung SCC in male patients. At the initial screening stage, Exiqon miRNA qPCR panels was utilized to conduct serum miRNA profile, which may be more sensitive and linear in measuring miRNAs with relatively low abundance compared to TaqMan platform. However, the results obtained from pooled samples may be inconsistent with the PCR results conducted on individual serum cases. Hence, our study performed three phases of qRT-PCR validation after the screening phase. In choosing the correct internal reference genes, geNorm was used to assess the most suitable internal reference control. Among the four tested genes, miR-16-5p had the least variation and relatively high levels of expression and was therefore considered as the most reliable gene for normalization. Whether miR-16-5p could be used as a reference molecule in the circulation is still controversial. It has been applied in many previous studies as a reference gene [19], however, it could also be secreted from and influenced by hemolysis [20]. Our result gave a strong support that miR-16-5p is stable in circulation and could be used as a reference gene. Three up-regulated miRNAs (miR106a-5p, miR-20a-5p and miR-93-5p) were identified and showed high accuracy in the diagnosis of lung SCC (AUC $=0.832$ ). The three-miRNA panel also possessed high accuracy in discriminating lung SCC from pulmonary hamartoma $(\mathrm{AUC}=0.900)$. In addition, when miR-103a$3 p$ was used for normalization, all of the three miRNAs
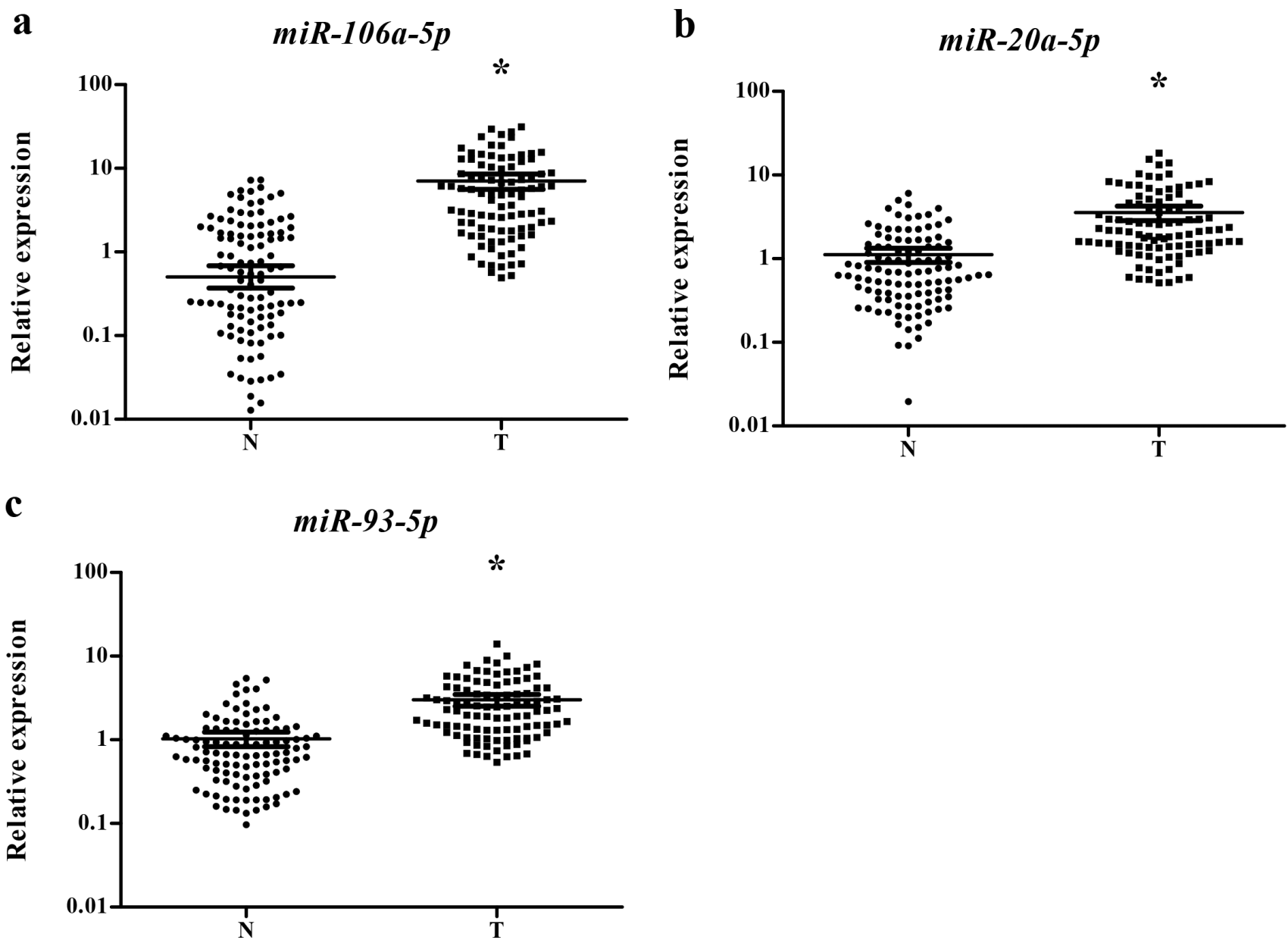

Figure 2: Expression levels of the three miRNAs in the serum of 102 lung SCC patients and $108 \mathrm{NCs}$. (a) miR-106a-5p; (b) miR-20a-5p; (c) miR-93-5p; N: normal controls; T: tumor. Horizontal line: mean with 95\% CI. ${ }^{\text {: }}$ P $<0.001$. 
were statistically significant (Supplementary Figure 5). Therefore, our study hypothesized that these miRNAs might be candidates for the noninvasive lung SCC detection in Chinese male patients.

Previous studies have identified a number of dysregulated serum miRNAs that could distinguish NSCLC patients from NCs. All of the three miRNAs identified in our study have been reported separately to have diagnostic power in NSCLC [21-24]. However, with the increasing understanding of the histologic and molecular differences among different subtypes of NSCLC, sub-grouping NSCLC into different subtypes for individualized treatment is essential both for safety and efficacy outcomes [25].
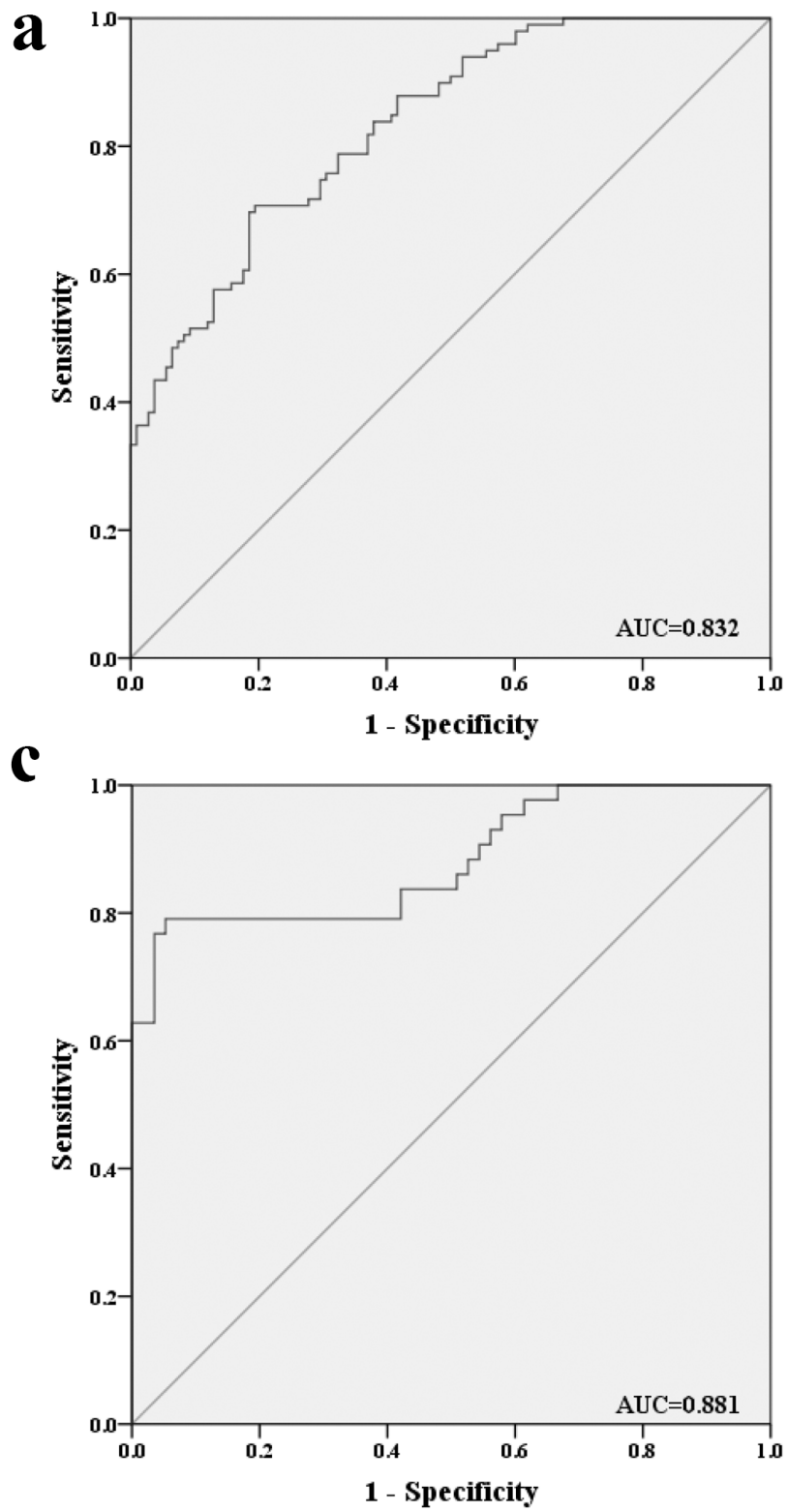

Recently, altered miRNA expression associated with the diagnosis of lung SCC has been reported by several studies. However, these studies mainly concentrated on tissue samples. The invasive procedures such as surgical section or biopsy to get tissue samples limit its application in the diagnosis of lung SCC. Only one previous study identified a five-miRNA signature (miR-205, miR-19a, miR-19b, miR-20a-5p, miR-451 and miR-30b) in the plasma of lung SCC patients that displayed significantly down-regulation after tumor resection [26]. Among the five miRNAs identified, serum miR-20a-5p was also confirmed in our study that could distinguish lung SCC from NCs. This provided evidence that there was uniformity between

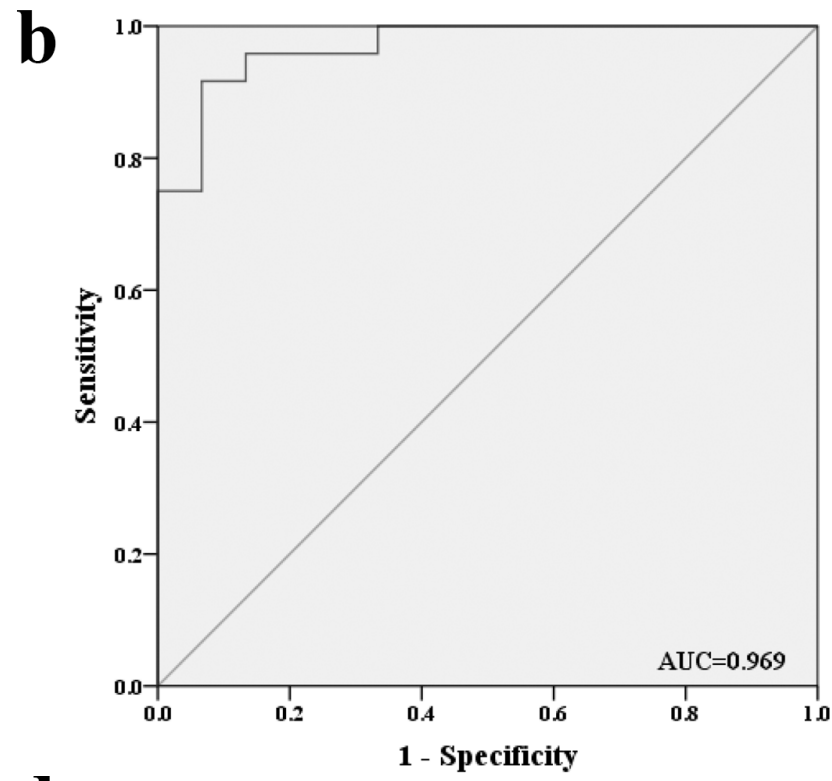

d

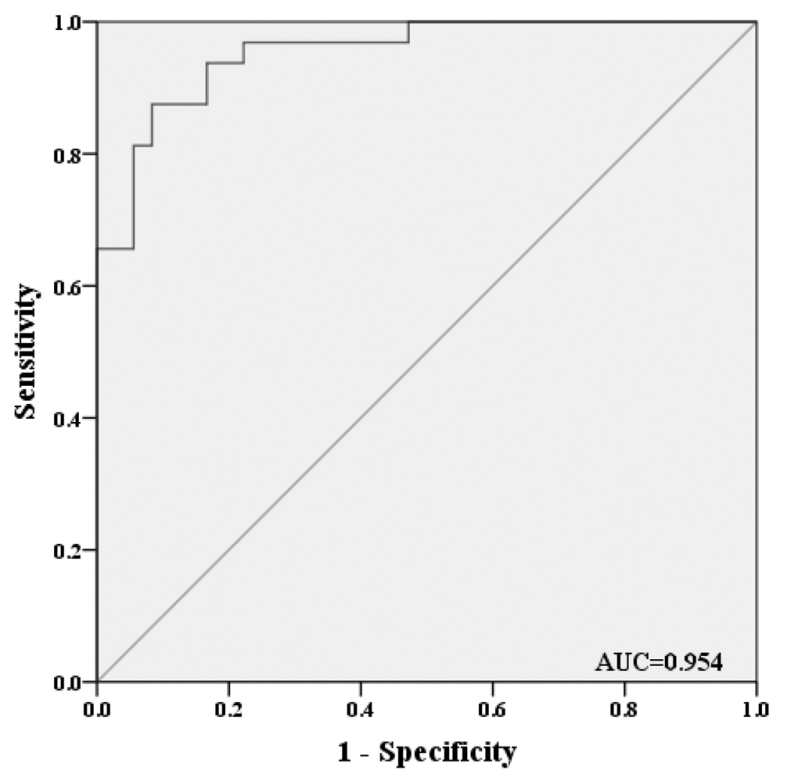

Figure 3: Receiver-operating characteristic (ROC) curves for the three-miRNA panel to discriminate lung SCC patients from NCs. (a) the combined three phases of training, testing and external validation phases (102 SCC VS. 108 NCs); (b) training phase (24 SCC VS. 15 NCs); (c) testing phase (44 SCC VS. 57 NCs). (d) external validation (34 SCC VS. 36 NCs). AUC: areas under the curve. 
$\mathbf{a}$

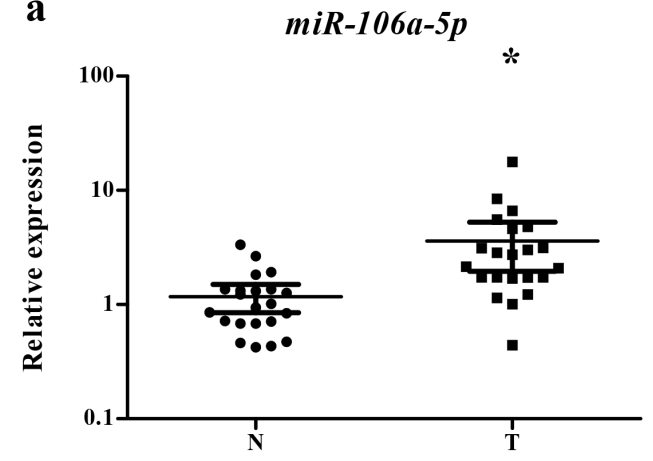

c

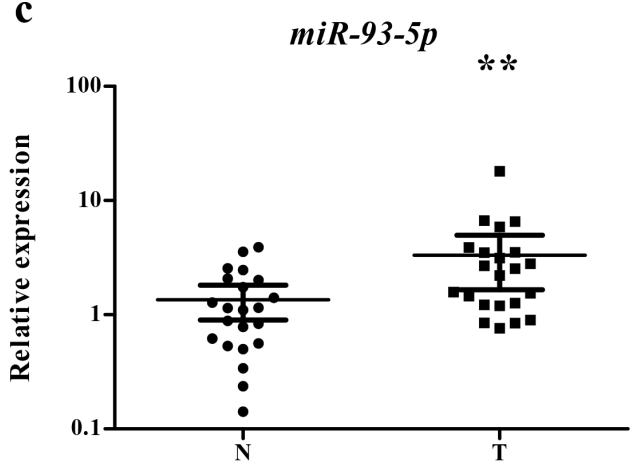

b

miR-20a-5p

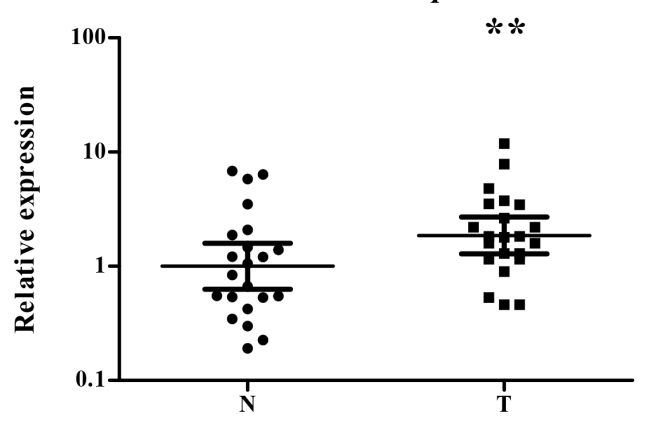

Figure 4: Expression of the three miRNAs in the tumor tissues of 23 pairs of lung SCC patients. (a) miR-106a-5p; (b) miR20a-5p; (c) miR-93-5p; N: normal controls; T: tumor. Horizontal line: mean with $95 \%$ CI. *: P $<0.001$; ${ }^{* *}: \mathrm{P}<0.05$.
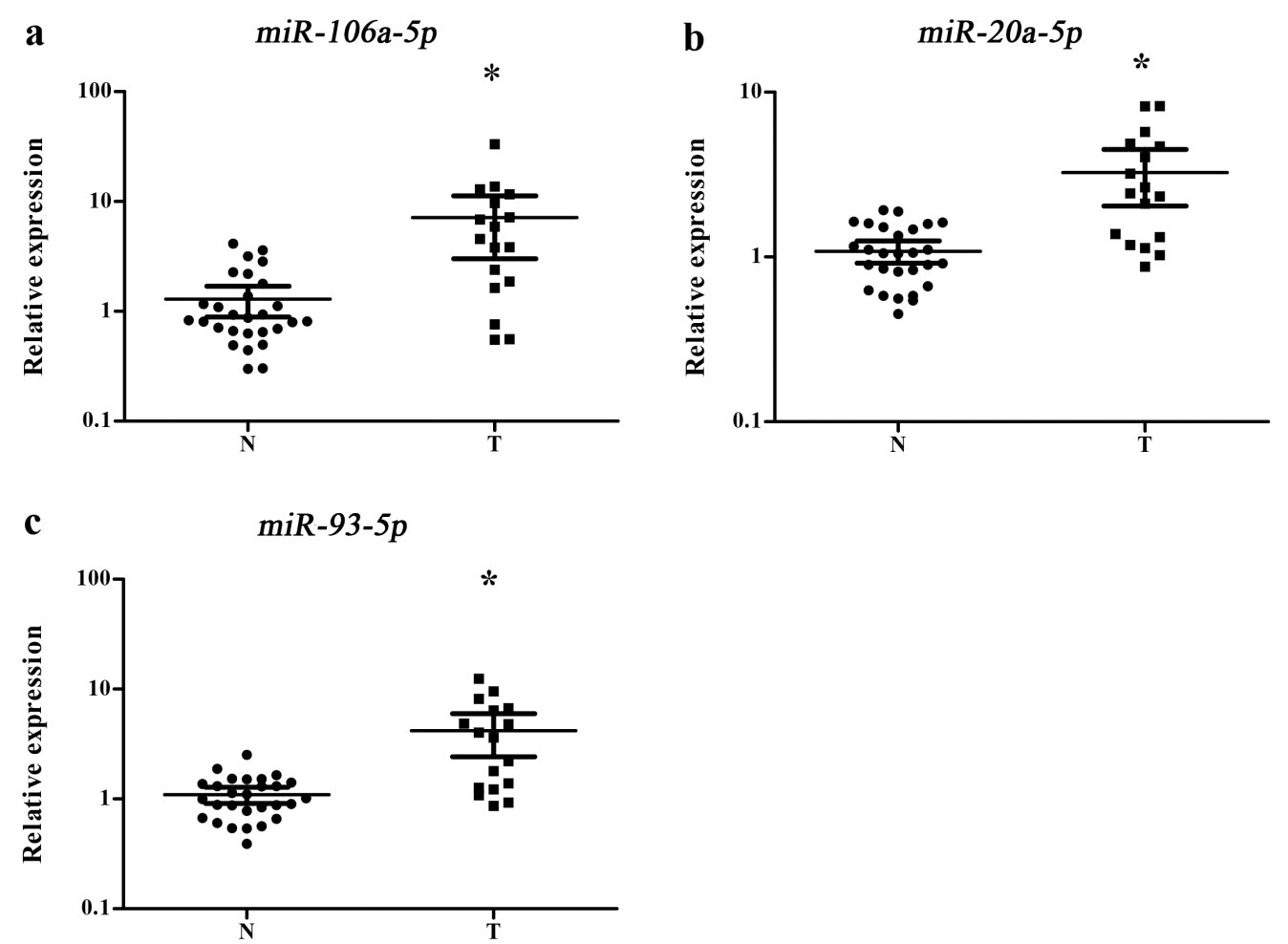

Figure 5: Expression of the three miRNAs in the serum exosomes of 17 lung SCC and 24 NCs. N: normal controls; T: tumor. : $: \mathrm{P}<0.001$. 
serum and plasma in the expression of miRNA. Elevated level of miR-20a-5p could promote growth and inhibit apoptosis in NSCLC cell lines by targeting T $\beta$ RII [27]. The expression of miR-20a-5p was upregulated in colorectal cancer based on The Cancer Genome Atlas (TCGA) project [28]. Meanwhile, as a member of the miR-17-92 cluster, circulating miR-20a-5p was proved to act as an oncogene in a wide range of other cancer types, such as colorectal cancer [28], gastric cancer [19], esophageal squamous cell carcinoma [29], nasopharyngeal carcinoma [30] as well as astrocytoma [31]. MiR-106a-5p is involved in cell proliferation and metastasis of NSCLC cell lines by targeting PTEN [32]. Up-regulation of miR-106a-5p could also enhance the chemoresistance of NSCLC cells to cisplatin by targeting $A B C A 1$ [33]. Moreover, miR-106a$5 \mathrm{p}$ was identified as an angiogenesis-related miRNA [34]. High levels of circulating miR-106a-5p were validated in metastatic breast cancer patients [35], gastric cancer [36] and colorectal cancer [37]. Based on TCGA data set, miR93-5p was identified to be a member of the pan-cancer oncogenic miRNA superfamily [38]. The overexpression of miR-93-5p could promote tumorigenesis by targeting tumor suppressor FUS1 [39] and DAB2 [40]. Meanwhile, it is able to inactivate ZNRF3 [41], leading to the activation of Wnt signaling, which promote cell proliferation and progression in lung cancer cell lines. Another target gene of miR-93-5p is LATS2, the reduced expression of LATS2 can result in enhanced angiogenesis and metastasis [42]. Elevated level of circulating miR-93-5p was identified as a diagnosis biomarker in ovarian cancer [43] and colorectal cancer [44].

The origin and secretory mechanism of circulating miRNAs remains a hot concern. Some studies believed that circulating miRNAs were generated from primary tumor tissues and then released into the blood [45, 46]. Thus, we verified the expression of the three miRNAs (miR-106a-5p, miR-20a-5p and miR-93-5p) in lung SCC tissues. All of the three miRNAs were up-regulated in tissue samples, which is in line with the theory. The mechanism behind the stability of circulating miRNAs is still unclear. Recent studies have revealed that miRNAs may be protected by forming complexes with proteins such as Argonaute and GW182 or in microvesicles or small membrane vesicles of endocytic origin called exosomes. Exosomes are 40-100 nm nano-sized vesicles that contain biologically active proteins and miRNAs [47]. Tumorderived exosmoes are emerging as important mediators of tumorigenesis and immune escape [48]. So, our study further explored exosomal serum miRNAs to identify the potential form of the three miRNAs in the extracellular environment. The expression of miR-106a-5p, miR-20a$5 p$ and miR-93-5p was all up-regulated in serum exosomes of lung SCC patients. Previously, the overexpression of serum exosomal miR-106a-5p and miR-20a-5p was reported to enhance cell proliferation and differentiation by down-regulating the MARK1 signaling pathway in nasopharyngeal carcinoma (NPC) [48]. The three identified miRNAs demonstrated a relatively abundant concentration in venous serum than that in arterial serum in our study. The phenomenon may be partly explained that hypoxia could increase the expression of the three miRNAs and may promote angiogenesis. Further studies are needed to find the exact mechanism.

Although the results are promising, a major limitation of our study was unable to detect the dynamic changes of serum miRNAs before and after surgery. Another limitation is that the number of patients enrolled was relatively small. Prospective studies on larger cohorts of patients are needed to confirm the diagnostic value of these miRNAs. In conclusion, our study identified a three-miRNA panel (miR-106a-5p, miR-20a-5p and miR-93-5p) in the serum of male lung SCC patients that could serve as an effective non-invasive biomarker for the diagnosis of lung SCC. Further studies involving larger cohorts are needed to validate the clinical application of these serum miRNAs.

\section{MATERIALS AND METHODS}

\section{Study design and study population}

Our study enrolled 102 patients who underwent lung tumor resection and 10 patients with pulmonary hamartoma at Jiangshu Cancer Hospital between 2012 and 2015. All the patients were male and histopathologically confirmed as lung SCC. 108 male normal controls (NCs) from the First Affiliated Hospital of Nanjing Medical University were acquired. The peripheral blood was drawn before surgery and none of the patients had received preoperative radiation or chemotherapy. Clinical and histopathological features of patients including age, smoking, TNM stage (according to the seventh edition American Joint Committee on Cancer (AJCC), differentiation degree were obtained retrospectively from the patients' records. Our study was approved by the institutional review board of Nanjing Medical University and the Hospital Ethics Committee. Written informed consent was obtained from every participant.

A multiphase study was designed to identify specific serum miRNAs for the diagnosis of lung SCC (Figure 1). In the initial screening stage, 30 serum samples from lung SCC patients and 10 from NCs were randomly selected and pooled as 3 lung SCC samples and 1 NC sample (10 samples were pooled as 1 sample). ExiqonmiRCURYReady-to-Use PCR-Human-panel-I+II-V1.M (Exiqon miRNA qPCR panel, Vedbaek, Denmark) were applied to identify miRNAs whose expression were altered in lung SCC samples compared to NCs as previously described. In the training stage, serum samples from 24 lung SCC patients and $15 \mathrm{NCs}$ were used to confirm the dysregulated miRNAs assessed by the screening stage. Subsequently in the testing stage, we refined the miRNAs by 44 lung SCC patients and 57 NCs. An external patient cohort of 34 lung SCC patients and $36 \mathrm{NCs}$ were used to assess the diagnostic value of the selected miRNAs. Additionally, another 10 patients with pulmonary hamartoma was used to validate 
the diagnostic performance of the candidate miRNAs. The panel of serum miRNAs was further validated in 23 pairs of tissue specimens and matched normal tissues. Serum exosomal miRNAs were also identified in 17 lung SCC patients and $28 \mathrm{NCs}$ to further discover the potential form of miRNAs in the peripheral blood. Arterial and venous blood samples from 3 lung SCC patients were obtained to compare the difference of miRNA expression between peripheral arterial and venous serum.

Venous blood samples of healthy controls and lung SCC patients without initial treatment were collected and placed in a serum separator tube. Cell-free serum was isolated from blood samples within 12 hours after collection using a two-step protocol (1,500 r.p.m. for $10 \mathrm{~min}, 12,000$ r.p.m. for $2 \mathrm{~min}$ ) to completely remove cell debris and then stored at $-80{ }^{\circ} \mathrm{C}$ until analysis. Tissue specimens were collected from surgery patients and kept in liquid nitrogen.

\section{Isolation of exosomes}

Exosomes were isolated from serum using ExoQuick $^{\mathrm{TM}}$ (System Biosciences, Mountain View, Calif) according to the manufacturer's protocol. Precipitated from $50 \mu 1$ ExoQuickexosome precipitation solutions and $200 \mu \mathrm{l}$ serum, exosome pellets were lysed in $200 \mu \mathrm{l}$ RNase-free water for further processing.

\section{RNA extraction}

Total RNA was extracted from $200 \mu \mathrm{l}$ serum or exosome using the mirVana PARIS Kit (Ambion, Austin, TX, USA) according to the manufacturer's protocol. $5 \mu l$ of synthetic C.elegans $m i R-39$ ( $5 \mathrm{nM} / \mathrm{L}$, RiboBio, Guangzhou, China) was added to each sample after the addition of denaturing solution (Ambion, Austin, TX, USA) for normalization. Trizol (Invitrogen, Carlsbad, CA, USA) was used to extract total RNA from tissue samples. Finally, total RNA was dissolved in $100 \mu \mathrm{l}$ RNasefree water and kept at $-80^{\circ} \mathrm{C}$ until further analysis. The ultraviolet spectrophotometer was applied to evaluate the concentration and purity of the extracted total RNA.

\section{Quantitative real-time polymerase chain reaction (qRT-PCR)}

The specific primers of reverse transcption (RT) and polymerase chain reaction (PCR) (RiboBio, China) were used to amplify miRNAs. The process of RT and PCR were performed as described previously [49-51]. MiRNAs were amplified and detected in a LightCycler 480 (Roche 480, Germany) real-time thermal cycler, using SYBR Green dye. The expression of miRNAs in serum and exosomes was calculated using the $2^{-\Delta \Delta \mathrm{Ct}}$ method relative to the combination of exogenous reference miRNA (cel$m i R-39)$ and endogenous reference miRNA (miR-16), $\Delta \mathrm{Ct}=$ CtmiRNA $-1 / 2$ (Ctcel-miR-39+CtmiR-16) [52]. The relative expression level of tissue miRNAs were calculated using the $2^{-\Delta \Delta \mathrm{Ct}}$ method and normalized to that of $R N U 6 B$ (U6).

\section{Statistical analysis}

GeNorm Version 3.5 was used to assess the expression stability of serum miRNA and the stability value (M) was calculated. Mann-Whitney test was used to analyze differential miRNAs expression between lung SCC patients and NCs. The association between miRNAs and the clinical characteristics was evaluated by $\chi 2$ test or paired-samples $t$ test. Receiver operating characteristic (ROC) curves and the area under the ROC curve (AUC) were used to estimate the diagnostic value of the candidate miRNAs for lung SCC. All the statistical analyses were performed using SPSS software (version 20.0, IBM, USA). A two-sided $P$ value $<0.05$ was considered statistically significant.

\section{Abbreviations}

miRNA, microRNA; NSCLC, Non-small cell lung cancer; SCC, squamous cell carcinoma; TCGA, The Cancer Genome Atlas; qRT-PCR, quantitative realtime polymerase chain reaction; $\mathrm{NC}$, normal control; ROC, receiver operating characteristic; LDCT: Low-dose computed tomography; survival; SCC Ag, squamous cell carcinoma antigen; NSE, neuron-specificenolase; AJCC, American Joint Committee on Cancer.

\section{CONFLICTS OF INTEREST}

The authors declare no competing financial interests.

\section{FUNDING}

This work was supported by the National Natural Science Foundation of China [Grant number: 81672400; 81672788; 81370516].

\section{REFERENCES}

1. Torre LA, Bray F, Siegel RL, Ferlay J, Lortet-Tieulent J, Jemal A. Global cancer statistics, 2012. CA Cancer J Clin. 2015; 65: 87-108.

2. Tanoue LT, Detterbeck FC. New TNM classification for non-small-cell lung cancer. Expert Rev Anticancer Ther. 2009; 9: 413-423.

3. Cancer Genome Atlas Research Network. Comprehensive genomic characterization of squamous cell lung cancers. Nature. 2012; 489: 519-525.

4. Derman BA, Mileham KF, Bonomi PD, Batus M, Fidler MJ. Treatment of advanced squamous cell carcinoma of the lung: a review. Transl Lung Cancer Res. 2015; 4: 524-532.

5. Sestini S, Boeri M, Marchiano A, Silva M, Calareso G, Galeone C, Sozzi G, Pastorino U. [Lung cancer screening in high-risk subjects: early detection with LDCT and risk 
stratification using miRNA-based blood test]. [Article in Italian]. Epidemiol Prev. 2016; 40: 42-50.

6. Song WA, Liu X, Tian XD, Wang W, Liang CY, Zhang T, Guo JT, Peng YH, Zhou NK. Utility of squamous cell carcinoma antigen, carcinoembryonic antigen, Cyfra 21-1 and neuron specific enolase in lung cancer diagnosis: a prospective study from China. Chin Med J (Engl). 2011; 124: 3244-3248.

7. Koberle V, Pleli T, Schmithals C, Augusto Alonso E, Haupenthal J, Bonig H, Peveling-Oberhag J, Biondi RM, Zeuzem S, Kronenberger B, Waidmann O, Piiper A. Differential stability of cell-free circulating microRNAs: implications for their utilization as biomarkers. PLoS One. 2013; 8: e75184.

8. Mitchell PS, Parkin RK, Kroh EM, Fritz BR, Wyman SK, Pogosova-Agadjanyan EL, Peterson A, Noteboom J, O'Briant KC, Allen A, Lin DW, Urban N, Drescher CW, et al. Circulating microRNAs as stable blood-based markers for cancer detection. Proc Natl Acad Sci U S A. 2008; 105: 10513-10518.

9. Mu YP, Sun WJ, Lu CW, Su XL. MicroRNAs may serve as emerging molecular biomarkers for diagnosis and prognostic assessment or as targets for therapy in gastric cancer. Asian Pac J Cancer Prev. 2015; 16: 4813-4820.

10. Liu R, Chen X, Du Y, Yao W, Shen L, Wang C, Hu Z, Zhuang R, Ning G, Zhang C, Yuan Y, Li Z, Zen K, et al. Serum microRNA expression profile as a biomarker in the diagnosis and prognosis of pancreatic cancer. Clin Chem. 2012; 58: 610-618.

11. Zheng G, Du L, Yang X, Zhang X, Wang L, Yang Y, Li J, Wang C. Serum microRNA panel as biomarkers for early diagnosis of colorectal adenocarcinoma. Br J Cancer. 2014; 111: 1985-1992.

12. Nadal E, Truini A, Nakata A, Lin J, Reddy RM, Chang AC, Ramnath N, Gotoh N, Beer DG, Chen G. A novel serum 4-microRNA signature for lung cancer detection. Sci Rep. 2015; 5: 12464.

13. Song J, Bai Z, Han W, Zhang J, Meng H, Bi J, Ma X, Han S, Zhang Z. Identification of suitable reference genes for qPCR analysis of serum microRNA in gastric cancer patients. Dig Dis Sci. 2012; 57: 897-904.

14. Shiotani A, Murao T, Kimura Y, Matsumoto H, Kamada T, Kusunoki H, Inoue K, Uedo N, Iishi H, Haruma K. Identification of serum miRNAs as novel non-invasive biomarkers for detection of high risk for early gastric cancer. Br J Cancer. 2013; 109: 2323-2330.

15. Mahdipour M, van Tol HT, Stout TA, Roelen BA. Validating reference microRNAs for normalizing qRT-PCR data in bovine oocytes and preimplantation embryos. BMC Dev Biol. 2015; 15: 25.

16. Zheng G, Wang H, Zhang X, Yang Y, Wang L, Du L, Li W, Li J, Qu A, Liu Y, Wang C. Identification and validation of reference genes for qPCR detection of serum microRNAs in colorectal adenocarcinoma patients. PLoS One. 2013; 8: e83025.
17. Charkiewicz R, Pilz L, Sulewska A, Kozlowski M, Niklinska W, Moniuszko M, Reszec J, Manegold C, Niklinski J. Validation for histology-driven diagnosis in non-small cell lung cancer using hsa-miR-205 and hsa-miR-21 expression by two different normalization strategies. Int J Cancer. 2016; 138: 689-697.

18. Liu X, Xie J, Liu Z, Gong Q, Tian R, Su G. Identification and validation of reference genes for quantitative RT-PCR analysis of retinal pigment epithelium cells under hypoxia and/or hyperglycemia. Gene. 2016; 580: 41-46.

19. Zhou X, Zhu W, Li H, Wen W, Cheng W, Wang F, Wu Y, Qi L, Fan Y, Chen Y, Ding Y, Xu J, Qian J, et al. Diagnostic value of a plasma microRNA signature in gastric cancer: a microRNA expression analysis. Sci Rep. 2015; 5: 11251.

20. Kirschner MB, Kao SC, Edelman JJ, Armstrong NJ, Vallely MP, van Zandwijk N, Reid G. Haemolysis during sample preparation alters microRNA content of plasma. PLoS One. 2011; 6: e24145.

21. Heegaard NH, Schetter AJ, Welsh JA, Yoneda M, Bowman ED, Harris CC. Circulating micro-RNA expression profiles in early stage nonsmall cell lung cancer. Int J Cancer. 2012; 130: 1378-1386.

22. Fan L, Qi H, Teng J, Su B, Chen H, Wang C, Xia Q. Identification of serum miRNAs by nano-quantum dots microarray as diagnostic biomarkers for early detection of non-small cell lung cancer. Tumour Biol. 2016; 37 : 7777-7784.

23. Leidinger P, Brefort T, Backes C, Krapp M, Galata V, Beier M, Kohlhaas J, Huwer H, Meese E, Keller A. Highthroughput qRT-PCR validation of blood microRNAs in non-small cell lung cancer. Oncotarget. 2016; 7: 4611-4623. https://doi.org/18632/oncotarget.6566.

24. Zhu W, He J, Chen D, Zhang B, Xu L, Ma H, Liu X, Zhang Y, Le H. Expression of miR-29c, miR-93, and miR-429 as potential biomarkers for detection of early stage non-small lung cancer. PLoS One. 2014; 9: e87780.

25. Langer CJ, Besse B, Gualberto A, Brambilla E, Soria JC. The evolving role of histology in the management of advanced non-small-cell lung cancer. J Clin Oncol. 2010; 28: 5311-5320.

26. Aushev VN, Zborovskaya IB, Laktionov KK, Girard N, Cros MP, Herceg Z, Krutovskikh V. Comparisons of microRNA patterns in plasma before and after tumor removal reveal new biomarkers of lung squamous cell carcinoma. PLoS One. 2013; 8: e78649.

27. Yang S, Cho YJ, Jin L, Yuan G, Datta A, Buckhaults P, Datta PK. An epigenetic auto-feedback loop regulates TGF-beta type II receptor expression and function in NSCLC. Oncotarget. 2015; 6: 33237-33252. https://doi. org/10.18632/oncotarget.4893.

28. Zekri AR, Youssef AS, Lotfy MM, Gabr R, Ahmed OS, Nassar A, Hussein N, Omran D, Medhat E, Eid S, Hussein MM, Ismail MY, Alenzi FQ, Bahnassy AA. Circulating serum miRNAs as diagnostic markers for colorectal cancer. PLoS One. 2016; 11: e0154130. 
29. He FC, Meng WW, Qu YH, Zhou MX, He J, Lv P, Ming L. Expression of circulating microRNA-20a and let-7a in esophageal squamous cell carcinoma. World $\mathrm{J}$ Gastroenterol. 2015; 21: 4660-4665.

30. Zeng X, Xiang J, Wu M, Xiong W, Tang H, Deng M, Li X, Liao Q, Su B, Luo Z, Zhou Y, Zhou M, Zeng Z, et al. Circulating miR-17, miR-20a, miR-29c, and miR-223 combined as non-invasive biomarkers in nasopharyngeal carcinoma. PLoS One. 2012; 7: e46367.

31. Zhi F, Shao N, Wang R, Deng D, Xue L, Wang Q, Zhang Y, Shi Y, Xia X, Wang S, Lan Q, Yang Y. Identification of 9 serum microRNAs as potential noninvasive biomarkers of human astrocytoma. Neuro Oncol. 2015; 17: 383-391.

32. Xie X, Liu HT, Mei J, Ding FB, Xiao HB, Hu FQ, Hu R, Wang MS. miR-106a promotes growth and metastasis of non-small cell lung cancer by targeting PTEN. Int J Clin Exp Pathol. 2015; 8: 3827-3834.

33. Ma Y, Li X, Cheng S, Wei W, Li Y. MicroRNA-106a confers cisplatin resistance in non-small cell lung cancer A549 cells by targeting adenosine triphosphatase-binding cassette A1. Mol Med Rep. 2015; 11: 625-632.

34. Cuevas A, Saavedra N, Cavalcante MF, Salazar LA, Abdalla DS. Identification of microRNAs involved in the modulation of pro-angiogenic factors in atherosclerosis by a polyphenol-rich extract from propolis. Arch Biochem Biophys. 2014; 557: 28-35.

35. Zhao Q, Deng S, Wang G, Liu C, Meng L, Qiao S, Shen L, Zhang Y, Lu J, Li W, Zhang Y, Wang M, Pestell RG, et al. A direct quantification method for measuring plasma MicroRNAs identified potential biomarkers for detecting metastatic breast cancer. Oncotarget. 2016; 7: 21865-21874. https://doi.org/10.18632/oncotarget.7990.

36. Hou X, Zhang M, Qiao H. Diagnostic significance of miR106a in gastric cancer. Int J Clin Exp Pathol. 2015; 8: 13096-13101.

37. Chen WY, Zhao XJ, Yu ZF, Hu FL, Liu YP, Cui BB, Dong XS, Zhao YS. The potential of plasma miRNAs for diagnosis and risk estimation of colorectal cancer. Int J Clin Exp Pathol. 2015; 8: 7092-7101.

38. Hamilton MP, Rajapakshe K, Hartig SM, Reva B, McLellan MD, Kandoth C, Ding L, Zack TI, Gunaratne PH, Wheeler DA, Coarfa C, McGuire SE. Identification of a pan-cancer oncogenic microRNA superfamily anchored by a central core seed motif. Nat Commun. 2013; 4: 2730.

39. Du L, Schageman JJ, Subauste MC, Saber B, Hammond SM, Prudkin L, Wistuba II, Ji L, Roth JA, Minna JD, Pertsemlidis A. miR-93, miR-98, and miR-197 regulate expression of tumor suppressor gene FUS1. Mol Cancer Res. 2009; 7: 1234-1243.

40. Du L, Zhao Z, Ma X, Hsiao TH, Chen Y, Young E, Suraokar M, Wistuba I, Minna JD, Pertsemlidis A. miR-93-directed downregulation of DAB2 defines a novel oncogenic pathway in lung cancer. Oncogene. 2014; 33: 4307-4315.
41. Shi J, Jiang X, Yu Z, He G, Ning H, Wu Z, Cai Y, Yu $\mathrm{H}$, Chen A. ZNRF3 contributes to the growth of lung carcinoma via inhibiting Wnt/beta-catenin pathway and is regulated by miR-93. Tumour Biol. 2016; 37: 3051-3057.

42. Fang L, Du WW, Yang W, Rutnam ZJ, Peng C, Li H, O'Malley YQ, Askeland RW, Sugg S, Liu M, Mehta T, Deng Z, Yang BB. MiR-93 enhances angiogenesis and metastasis by targeting LATS2. Cell Cycle. 2012; 11: 4352-4365.

43. Meng X, Joosse SA, Muller V, Trillsch F, Milde-Langosch K, Mahner S, Geffken M, Pantel K, Schwarzenbach H. Diagnostic and prognostic potential of serum miR-7, miR-16, miR-25, miR-93, miR-182, miR-376a and miR429 in ovarian cancer patients. Br J Cancer. 2015; 113: 1358-1366.

44. Wang S, Xiang J, Li Z, Lu S, Hu J, Gao X, Yu L, Wang L, Wang J, Wu Y, Chen Z, Zhu H. A plasma microRNA panel for early detection of colorectal cancer. Int J Cancer. 2015; 136: $152-161$.

45. Wang K, Zhang S, Marzolf B, Troisch P, Brightman A, Hu Z, Hood LE, Galas DJ. Circulating microRNAs, potential biomarkers for drug-induced liver injury. Proc Natl Acad Sci U S A. 2009; 106: 4402-4407.

46. Brase JC, Johannes M, Schlomm T, Falth M, Haese A, Steuber T, Beissbarth T, Kuner R, Sultmann H. Circulating miRNAs are correlated with tumor progression in prostate cancer. Int J Cancer. 2011; 128: 608-616.

47. Ma R, Jiang T, Kang X. Circulating microRNAs in cancer: origin, function and application. J Exp Clin Cancer Res. 2012; 31: 38 .

48. Ye SB, Li ZL, Luo DH, Huang BJ, Chen YS, Zhang XS, Cui J, Zeng YX, Li J. Tumor-derived exosomes promote tumor progression and T-cell dysfunction through the regulation of enriched exosomal microRNAs in human nasopharyngeal carcinoma. Oncotarget. 2014; 5: 5439-5452. https://doi. org/10.18632/oncotarget.2118.

49. Li H, Zhou X, Zhu J, Cheng W, Zhu W, Shu Y, Liu P. MiR4728-3p could act as a marker of HER2 status. Cancer Biomark. 2015; 15: 807-814.

50. Zhao DS, Chen Y, Jiang H, Lu JP, Zhang G, Geng J, Zhang Q, Shen JH, Zhou X, Zhu W, Shan QJ. Serum miR-210 and miR-30a expressions tend to revert to fetal levels in Chinese adult patients with chronic heart failure. Cardiovasc Pathol. 2013; 22: 444-450.

51. Qiu T, Zhou X, Wang J, Du Y, Xu J, Huang Z, Zhu W, Shu Y, Liu P. MiR-145, miR-133a and miR-133b inhibit proliferation, migration, invasion and cell cycle progression via targeting transcription factor $\mathrm{Sp} 1$ in gastric cancer. FEBS Lett. 2014; 588: 1168-1177.

52. Sourvinou IS, Markou A, Lianidou ES. Quantification of circulating miRNAs in plasma: effect of preanalytical and analytical parameters on their isolation and stability. J Mol Diagn. 2013; 15: 827-834. 